\title{
Coupling of plasticity and damage in glass fibre reinforced polymer composites
}

\author{
R. Kvale Joki ${ }^{1,2, a}$, F. Grytten ${ }^{1}$, and H. Osnes ${ }^{2}$ \\ ${ }^{1}$ SINTEF Materials \& Chemistry \\ ${ }^{2}$ University of Oslo, Applied Mathematics and Mechanics
}

\begin{abstract}
This study addresses the nonlinear stress-strain response in glass fibre reinforced polymer composite laminates. Loading and unloading of these laminates indicate that the nonlinear response is caused by both damage and plasticity. A user defined material model is implemented in the finite element code LS-DYNA. The damage evolution is based on the Puck failure criterion [1], and the plastic behaviour is based on the quadratic Hill yield criterion for anisotropic materials [2].
\end{abstract}

\section{Introduction}

A major challenge in composite design is to calculate the damage state and the residual strength after failure initiation. Composite materials have been thought to be brittle with little or no ability for plastic deformation [1]. Non-linear stress-strain response has therefore been treated as damage. Here damage refers to the more or less gradual developments of micro cracks in the matrix material which lead to macro cracks and then finally to ultimate failure caused by fibre failure.

In experiments conducted on laminated glass fibre reinforced polymer composites (GFRPCs) it has been shown that when the specimens were unloaded after tensile loading, irreversible strains had emerged. This indicates that non-linearity is not only caused by damage, but also by plasticity. When loaded a second time, the stress-strain curve also showed some hysteresis, indicating visco-elastic behaviour of the composites.

It is known that composite materials are sensitive to impact loading. A key aspect of an impact event is the impact energy. If one is to understand how the impact energy is absorbed, it is crucial to understand the material response. A non-linear stress-strain response caused by elastic-plastic deformation with irreversible strains absorbs more energy than the equivalent response caused by elastic-damage deformation without irreversible strains, since the dissipated energy equals the area circumfered by the stress-strain curve, see Figure 2 and 3. A presence of hysteresis in the cyclic loading may also serve to dissipate some amount of energy if the propagation of shock waves trough the material causes a large number of oscillations in the local state of stress.

To evaluate the damage progression a material model based on the Puck failure criterion has been implemented in the LS-DYNA finite element code. The Puck criterion [1] was ranked as one of the most promising in the World Wide Failure Exercises (WWFE) [4]. One of the conclusion from the WWFE was that the criteria should treat fibre failure and matrix failure separately. For progressive failure analysis this is a crucial aspect since fibre failure and matrix failure evolve quite differently. The

a e-mail: reidar.joki@sintef.no
Puck criterion differentiates between Fibre failure (FF) and Inter Fibre failure (IFF). The FF criterion evaluate fibre failure in compression and tension, and the IFF criterion evaluates all failure mechanisms associated with the matrix in compression, tension and shear. The criterion has separate expressions for tension and compression for both FF and IFF. When the conditions defined by the FF criterion are reached in an integration point, the material model reduces the stiffness in that location to zero by deleting the element containing that integration point. When the conditions defined by the IFF criterion are present in an integration point, on the other hand, the material model reduces the stiffness in that integration point separately, representing the softening caused by the progression of a smeared damage.

To include plastic strain, the quadratic Hill yield criterion has also been implemented in the LS-DYNA finite element code. The quadratic Hill yield criterion for anisotropic materials [2] is an extension the von Mises criterion for isotropic materials, containing six parameters specifying the state of anisotropy. When describing transversely isotropic materials, four parameters are sufficient.

The evolution of both damage and plasticity is based on the nonlinear response calculated from tensile testing of various laminates. It is assumed that the global nonlinearity is caused by mechanisms in the matrix material alone, and that the fibres behave linearly elastic until sudden failure. The stress-strain curves from standard tensile tests can be replicated by pure damage models as well as pure plastic models, but when the specimens are unloaded after straining the response can not be replicated by damage or plasticity alone. The actual response call for a model based on both damage and plasticity.

The tested laminates are made of standard E-glass fibres embedded in an epoxy based vinylester matrix ${ }^{1}$. The laminates are made of vacuum infused non crimp fabric. Since the laminates are laid up as non crimp fabric, the unidirectional laminates have $5 \%$ of the fibre weight fraction in the transverse direction. When modeling these layers it is assumed that the fibres in the transverse direction can be evaluated as distinct layers with the same volume fraction as the fibres in the longitudinal direction. 


\section{The Puck failure criterion}

The Puck failure criterion is applicable to composite laminates built up of unidirectional plies. The plies are assumed to be transversely isotropic and the anisotropy is defined by Cartesian coordinate axes, with 1-direction being in the direction parallel to the fibres, 2-direction being the orthogonal in-plane direction, and the 3-direction the ply normal. The plane of isotropy is defined by the 2- and 3-axes.

\subsection{Fibre failure}

It is assumed that there exists a perfect adhesion between fibre and matrix, i.e. the strain parallel to the fibres $\left(\epsilon_{11}\right)$ are the same for both fibres $\left(\epsilon_{\mathrm{f} 1}\right)$ and matrix $\left(\epsilon_{\mathrm{m} 1}\right)$ : $\epsilon_{\mathrm{f} 1}=\epsilon_{11}$. Under combined loading the strain in the fibres will be: $\epsilon_{\mathrm{f} 1}=\frac{\sigma_{\mathrm{f} 1}}{E_{\mathrm{f} 1}}-\frac{v_{\mathrm{f} 12}}{E_{\mathrm{f} 1}} m_{\sigma \mathrm{f}} \sigma_{22}$, where $v_{\mathrm{f} 12}$ is the Poisson's ratio for the fibres, and $m_{\sigma \mathrm{f}}$ accounts for the stress magnification effect ${ }^{2}$ caused by the different moduli of matrix and fibres, which leads to an uneven distribution of $\sigma_{22}$ from a microscopic point of view. Thus the longitudinal stress in the fibres $\left(\sigma_{\mathrm{f} 1}\right)$ under combined loading is defined as: $\sigma_{\mathrm{f} 1}=\epsilon_{11} E_{\mathrm{f} 1}+v_{\mathrm{f} 12} m_{\sigma \mathrm{f}} \sigma_{22}$. Therefore the failure condition for fibre failure under combined loading can be described as:

$$
\frac{1}{\epsilon_{1 T}}\left(\epsilon_{11}+\frac{v_{\mathrm{f} 12}}{E_{\mathrm{f} 1}} m_{\sigma \mathrm{f}} \sigma_{2}\right)=1 \text { for }(\ldots) \geqslant 1,
$$

were $\epsilon_{1 T}$ is the ultimate tensile strain in the longitudinal direction. Experimental results have shown that when a significant shear stress, $\tau_{12}$, is superimposed, the compressive strength is significantly reduced. Puck has therefore added a shear correction factor to the criterion for compressive load:

$$
\frac{1}{\epsilon_{1 \mathrm{C}}}\left|\left(\epsilon_{11}+\frac{v_{\mathrm{f} 12}}{E_{\mathrm{f} 1}} m_{\sigma \mathrm{f}} \sigma_{22}\right)\right|+\left(10 \gamma_{21}\right)^{2}=1 \text { for }(\ldots)<1,
$$

where $\epsilon_{1 C}$ is the ultimate compressive strain in the longitudinal direction, and $\left(10 \gamma_{21}\right)^{2}$ is a purely empirical term. It is preferable to use an expression with $\gamma_{21}$ instead of $\tau_{21}$, because the value of $\tau_{21}$ is unknown after failure initiation, whereas the value of $\gamma_{21}$ is not.

\subsection{Inter fibre failure}

The Puck failure criterion has an analytical solution for in-plane loading and failure conditions [1] (in two dimensional analysis). When taking into account out of plane effects, i.e. trough the thickness shear variations, and loading conditions in three dimensions, the Puck IFF criterion has no analytical solution. A full 3-D formulation of the Puck IFF criterion was used by Wigand et al. [5],

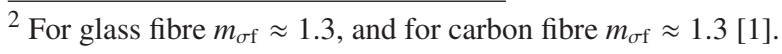

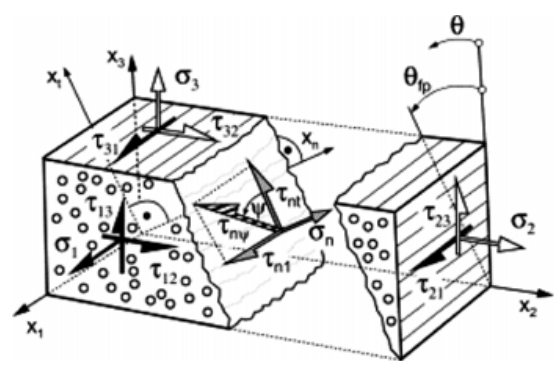

Fig. 1. Illustration of the fracture surface and the stress components acting on the surface [1].

and is formulated as:

$$
\begin{aligned}
e^{2} & =\left(\frac{\sigma_{\mathrm{n}}}{R_{\mathrm{n}}}\right)^{2}+\left(\frac{\tau_{\mathrm{n} 1}}{R_{\mathrm{n} 1}-p_{\mathrm{n} 1} \sigma_{\mathrm{n}}}\right)^{2}+\left(\frac{\tau_{\mathrm{nt}}}{R_{\mathrm{nt}}-p_{\mathrm{nt}} \sigma_{\mathrm{n}}}\right)^{2} \\
& =1 \text { for } \sigma_{\mathrm{n}} \geqslant 0 \\
e^{2} & =\left(\frac{\tau_{\mathrm{n} 1}}{R_{\mathrm{n} 1}-p_{\mathrm{n} 1} \sigma_{\mathrm{n}}}\right)^{2}+\left(\frac{\tau_{\mathrm{nt}}}{R_{\mathrm{nt}}-p_{\mathrm{nt}} \sigma_{\mathrm{n}}}\right)^{2} \\
& =1 \text { for } \sigma_{\mathrm{n}}<0
\end{aligned}
$$

where the stress components $\sigma_{\mathrm{n}}, \tau_{\mathrm{n} 1}$ and $\tau_{\mathrm{nt}}$ are the stresses acting on the fracture surface as illustrated in Figure 1 and defined by the angle of the fracture surface, $\theta_{\mathrm{fp}}$, in Equation (5). The parameter $R_{\mathrm{n}}$ describes the resistance of the fracture plane against normal failure induced by $\sigma_{\mathrm{n}}$. The parameters $R_{\mathrm{n} 1}$ and $R_{\mathrm{nt}}$ are the resistance of the fracture plane against shear, and finally, $p_{\mathrm{n} 1}$ and $p_{\mathrm{nt}}$ are the slope parameters representing internal friction effects [5]. Puck suggests the following values for these parameters [6]

$$
\begin{aligned}
R_{\mathrm{n}} & =S_{22}^{+}, \\
R_{\mathrm{n} 1} & =S_{12}, \\
R_{\mathrm{nt}} & =\frac{S_{22}^{-}}{2 \tan \left(\theta_{\mathrm{fp}}^{0}\right)}, \\
p_{\mathrm{nt}} & =-\frac{1}{\tan \left(2 \theta_{\mathrm{fp}}^{0}\right)}, \\
p_{\mathrm{n} 1} & =p_{\mathrm{nt}} \frac{R_{\mathrm{n} 1}}{R_{\mathrm{nt}}} .
\end{aligned}
$$

Here, $Y_{t}$ and $Y_{c}$ are the transverse strengths in tension and compression, respectively, and $S_{12}$ is the shear strength. The angle $\theta_{\mathrm{fp}}^{0}$ is the angle at which a specimen loaded in uniaxial compression in the transverse fibre direction fracture due to shear failure. When subjected to in-plane loading, IFF is provoked by $\sigma_{22}$ and $\tau_{12}$. The shape of the failure envelope depends strongly on $\theta_{\mathrm{fp}}^{0}$, especially for the interpolation between in-plane shear and compressive transverse loading. This angle can be viewed upon as a material property and has a constant value for a given material. It should be emphasized that this is not the same angle as the fracture angle defining the action plane of an arbitrary loading condition $\theta_{\mathrm{fp}}$. The stresses acting on the failure surface are depend on the angle of the failure 
surface, and are calculated as:

$$
\begin{aligned}
\sigma_{\mathrm{n}} & =\sigma_{22} \cos ^{2} \theta_{\mathrm{fp}}+\sigma_{33} \sin ^{2} \theta_{\mathrm{fp}} \\
& +2 \sigma_{23} \cos \theta_{\mathrm{fp}} \sin \theta_{\mathrm{fp}}, \\
\tau_{\mathrm{n} 1} & =\sigma_{12} \cos \theta_{\mathrm{fp}}+\sigma_{13} \sin ^{2} \theta_{\mathrm{fp}}, \\
\tau_{\mathrm{nt}} & =-\sigma_{22} \sin \theta_{\mathrm{fp}} \cos \theta_{\mathrm{fp}}+\sigma_{33} \sin \theta_{\mathrm{fp}} \cos \theta_{\mathrm{fp}} \\
& +\sigma_{23}\left(\cos ^{2} \theta_{\mathrm{fp}}-\sin ^{2} \theta_{\mathrm{fp}}\right) .
\end{aligned}
$$

Because no analytical solution to the criterion is possible, one needs to search through all the possible failure surfaces to find the one in where the criterion has its maximum value. The criterion is defined by the failure surface stress components $\sigma_{\mathrm{n}}, \tau_{\mathrm{n} 1}$ and $\tau_{\mathrm{nt}}$, and consequently they have to be calculated for every search angle. Because of symmetry, the search for the action plane can be limited to the range $-90^{\circ} \geqslant \theta \geqslant 90^{\circ}$. A cost effective methodology is the Golden Section Search [5]. This procedure has been proven to be an effective tool when searching for the action plane using the Puck criterion by Wiegand et al. [5].

The strength parameters found in the IFF criterion $\left(S_{22}^{+}, S_{22}^{-}\right.$and $\left.S_{12}\right)$ represents the end of the linearity in the stress-strain response. The reasons for this non-linearity are plastic and viscoelastic behaviour of the matrix and, probably above all, micro-damage [7]. Typically a micro crack in the matrix propagate until it is stopped by a crossing fibre. At the location of the micro-damage, the stiffness of the matrix is reduced to zero, while the rest of the matrix material holds the initial stiffness. As more and more of these micro cracks develops, the overall global stiffness will be degraded in a progressive manner. The development of these micro-cracks will affect the global transverse stiffnesses $E_{22}$ and $E_{33}$, the in- and out-of-plane shear stiffness's $G_{12}$ and $G_{13}$, the transverse out-of-plane shear stiffness $G_{23}$, the in- and out-off-plane Poisson's ratio $v_{12}$ and $v_{13}$ and the transverse out-off-plane Poisson's ratio $v_{23}$. It is reasonable to address the development of micro-damage as smeared, and degrade the $E_{22}, E_{33}, G_{12}$, $G_{13}, v_{12}, v_{13}$ and $v_{23}$ with a progressive reduction factor. In [7], Puck argues that these material properties may be reduced concurrently by the same reduction factor, $\eta$. Puck also argues that the following expression is a reasonable approach to this reduction factor [7]:

$$
\eta=\frac{1-\eta_{\mathrm{r}}}{1+c\left(f_{\mathrm{E}(\mathrm{IFF})}-1\right)^{\xi}}+\eta_{\mathrm{r}}
$$

where the dimensionless parameters $c$ and $\xi$ serve to fit the $\eta$-curve to experimental results. The term $\eta_{r}$ represents a small remaining stiffness $\left(\eta_{\mathrm{r}}<1\right)$. The values of $c, \xi$ and $\eta_{\mathrm{r}}$ has been determined using the optimization code LS-OPT. The simulated response has been fitted to experimental results from tensile loading of 6-layered laminates with 5\% of the fibres in each layer in the longitudinal direction and $95 \%$ in the transverse direction. It is assumed that the $5 \%$ of the fibres that are oriented in the longitudinal direction behave elastic untill failure.

Figure 2 illustrates the on and off loading response of a $[ \pm 45]_{6}$-laminate when nonlinearity is caused by damage alone. The laminate is loaded and unloaded at point 1 , 2 and 3. The unloading follows a linear-elastic path to zero stress and strain. When the laminate is resloaded, the

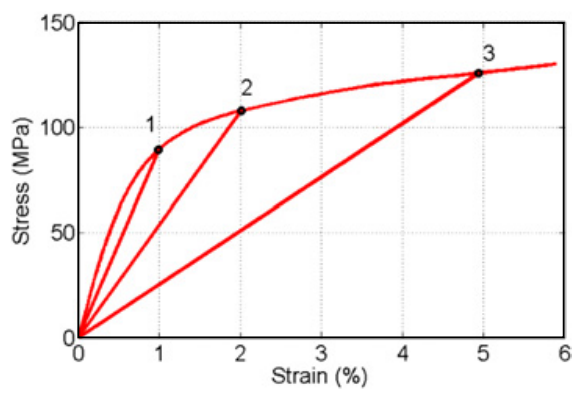

Fig. 2. Three cycles of loading and unloading of $[ \pm 45]_{6^{-}}$ laminates.

stress-strain curve follows the same path as when unloaded until it succeedes the stress level of the previous unloaded. As the stress level is increased, further damage is induced by continued reduction of the secant stiffness.

\section{The Hill yield criterion}

The original Hill yield criterion [2] has a quadratic form, and is a straightforward extension of the von Mises yield criterion. It is defined by three axes of anisotropy.

$$
\begin{aligned}
\bar{\sigma}^{2}= & F\left(\sigma_{22}-\sigma_{33}\right)^{2}+G\left(\sigma_{33}-\sigma_{11}\right)^{2} \\
& +F\left(\sigma_{11}-\sigma_{22}\right)^{2}+2 L \sigma_{23}^{2} \\
& +2 M \sigma_{31}^{2}+2 N \sigma_{12}^{2} .
\end{aligned}
$$

Here $F, G, H, L, M$ and $N$ are constants that have to be determined experimentally, $\sigma_{i j}$ are the stresses with reference to the axes of anisotropy and $\bar{\sigma}$ is the effective stress. For composite laminates made up of unidirectional plies, the axes of anisotropy are orthogonal. The constants can be determined from [2]:

$$
\begin{aligned}
& F=\frac{1}{2}\left[\left(\frac{\sigma_{0}}{\sigma_{22}^{y}}\right)^{2}+\left(\frac{\sigma_{0}}{S_{33}^{y}}\right)^{2}-\left(\frac{\sigma_{0}}{S_{11}^{y}}\right)^{2}\right], \\
& G=\frac{1}{2}\left[\left(\frac{\sigma_{0}}{S_{33}^{y}}\right)^{2}+\left(\frac{\sigma_{0}}{S_{11}^{y}}\right)^{2}-\left(\frac{\sigma_{0}}{S_{22}^{y}}\right)^{2}\right], \\
& H=\frac{1}{2}\left[\left(\frac{\sigma_{0}}{S_{11}^{y}}\right)^{2}+\left(\frac{\sigma_{0}}{S_{22}^{y}}\right)^{2}-\left(\frac{\sigma_{0}}{S_{33}^{y}}\right)^{2}\right]
\end{aligned}
$$

and

$$
\begin{aligned}
L & =\frac{1}{2}\left(\frac{\sigma_{0}}{S_{23}^{y}}\right)^{2}, \\
M & =\frac{1}{2}\left(\frac{\sigma_{0}}{S_{31}^{y}}\right)^{2}, \\
N & =\frac{1}{2}\left(\frac{\sigma_{0}}{S_{12}^{y}}\right)^{2},
\end{aligned}
$$

where $\sigma_{0}$ is the effective stress at initiation of plasticity. $S_{i j}^{y}$, for $i=j$, are the normal yield strengths with respect to the axes of anisotropy, and $S_{i j}^{y}$, for $i \neq j$, are the shear yield strengths with respect to the axes of anisotropy. 


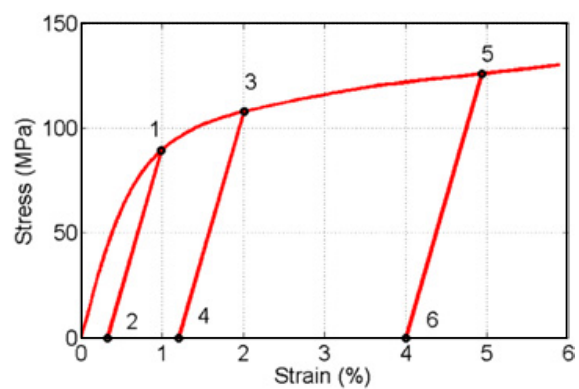

Fig. 3. Three cycles of loading and unloading of the $[ \pm 45]_{6^{-}}$ laminates.

As mentioned above, it is assumed that the plane orthogonal to the fibre direction is isotropic. By this assumption of transverse isotropy it follows that $S_{22}=S_{33}$ and $S_{12}=S_{13}$, which again serves to reduce the number of constants from six to four, i.e. $G=H$ and $M=N$. Therefore the quadratic Hill yield criterion can be reduced to:

$$
\begin{aligned}
\bar{\sigma}^{2}= & F\left(\sigma_{22}-\sigma_{33}\right)^{2} \\
& +G\left[\left(\sigma_{33}-\sigma_{11}\right)^{2}+\left(\sigma_{11}-\sigma_{22}\right)^{2}\right] \\
& +2 L \sigma_{23}^{2}+2 M\left[\sigma_{13}^{2}+\sigma_{12}^{2}\right] .
\end{aligned}
$$

Figure 3 illustrates the on and off loading response of a $[ \pm 45]_{6}$-laminate when nonlinearity is assumed to be caused by plasticity alone. The laminate is loaded and unloaded at the same strain levels as the laminate described in 2, at 1,2 and $5 \%$ strain. When the nonlinearity is described by plasticity, the nonlinearity is achieved by introducing irreversible strains. This is evident when the laminate is unloaded and the stress level is reduced to zero, the strain level is non-zero. It can be seen that the on and off loading paths 1-2, 3-4 and 5-6, have the same gradient, indicating that the global stiffness of the laminate is preserved.

\section{Material tests}

All the material properties used to describe the elastic behaviour are based on an extensive material testing exercise using low strain rates. The tensile tests are carried out in accordance to the ASTM D3039 standard, the compressive tests, to the ASTM D6641 and the shear properties are obtained from ASTM D 3518 and ASTM D7078. Seven different laminate lay-ups have been tested $[0]_{6},[90]_{6}$, $[0 / 90]_{6},[ \pm 45]_{6},[0 / 45 / 90 /-45]_{6},\left[ \pm 45 /(0)_{6} / \pm 45\right]$ and $\left[ \pm 45 /(90)_{6} / \pm 45\right]$, where the numbers within the brackets indicate the orientation of the longitudinal ply direction with respect to the loading direction, the order of the numbers indicate the stacking sequence and the subscript out side the brackets, how many times the sequence is repeated through the laminate. The deduced elastic material properties are displayed in Table 1 and the strength parameters (with respect to damage) are displayed in Table 2. The parameters needed to evaluate the Puck failure criterion are displayed in Table 3, here the fracture angle for pure transverse compression, $\theta_{\mathrm{fp}}$, is obtained from literature for
Table 1. Elastic material properties.

\begin{tabular}{ll}
\hline \hline$E_{11}$ & $42028 \mathrm{MPa}$ \\
$E_{22}$ & $11472 \mathrm{MPa}$ \\
$G_{12}$ & $4000 \mathrm{MPa}$ \\
$v_{12}$ & 0.25 \\
$v_{23}$ & 0.30 \\
\hline
\end{tabular}

Table 2. Material strength.

\begin{tabular}{ll}
\hline \hline$S_{11}^{+}$ & $1100 \mathrm{MPa}$ \\
$S_{22}^{+}$ & $28 \mathrm{MPa}$ \\
$S_{11}^{-}$ & $600 \mathrm{MPa}$ \\
$S_{22}^{-}$ & $200 \mathrm{MPa}$ \\
$S_{12}$ & $50 \mathrm{MPa}$ \\
$S_{23}$ & $50 \mathrm{MPa}$ \\
\hline
\end{tabular}

Table 3. Properties for the Puck criterion.

\begin{tabular}{ll}
\hline \hline$\theta_{f p}^{0}$ & 51 \\
$E_{1 f}^{+}$ & $73000 \mathrm{MPa}$ \\
$v_{1 f}$ & 0.20 \\
\hline
\end{tabular}

Table 4. Properties for the Hill criterion.

\begin{tabular}{ll}
\hline \hline$F$ & 0.9997 \\
$G$ & $3.24 \mathrm{E}-05$ \\
$L$ & 0.160 \\
$M$ & 0.160 \\
\hline
\end{tabular}

an equivalent material. The plasticity parameters needed to evaluate the Hill criterion are calculated as described above, and displayed in Table 4.

Two sets of laminates have been subjected to cycles of loading and unloading, namely $[0 / 90]_{6^{-}}$and $[ \pm 45]_{6^{-}}$ laminates. The strain rate and on- and off-loading tests has been conducted using the setup and test specimen geometry from the ASTM D 3039 test standard. The results are presented in Figure 4 and 5. It is assumed that the longitudinally oriented plies in the $[0 / 90]_{6}$-laminates behave elastically within the $2.5 \%$ strain. This assumption is based on an evaluation of the stress-strain curves produced from tensile tests on the various laminates mentioned above. Therefore the nonlinearity is thought to be caused by mechanisms in the transversely oriented plies. In Figure 6 the response from the transversely oriented plies has been isolated using classical laminate theory (the Poisson effects are disregarded).

The unloading and reloading paths observed in the $[ \pm 45]_{6}$-laminates indicate that the initial stiffness is only slightly reduced. It is therefore assumed that the nonlinearity is mainly caused by plasticity, whereas the tensile response in the transverse direction is mainly caused by 


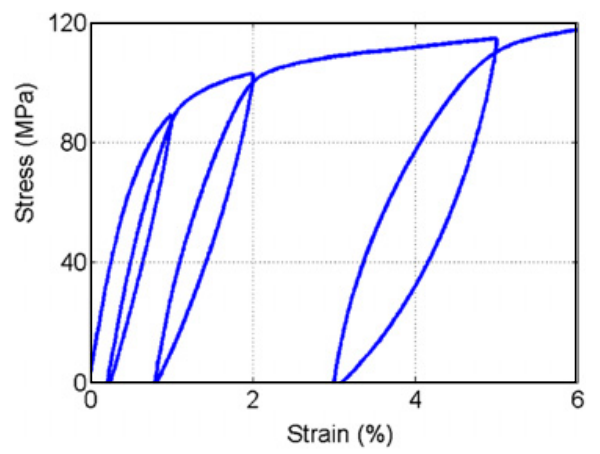

Fig. 4. Three cycles of loading and unloading of the $[ \pm 45]_{6}$ laminates.

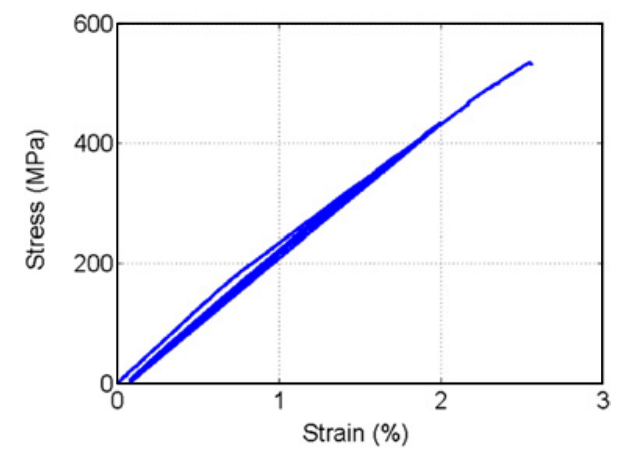

Fig. 5. Three cycles of loading and unloading of the $[0 / 90]_{6}$ laminates.

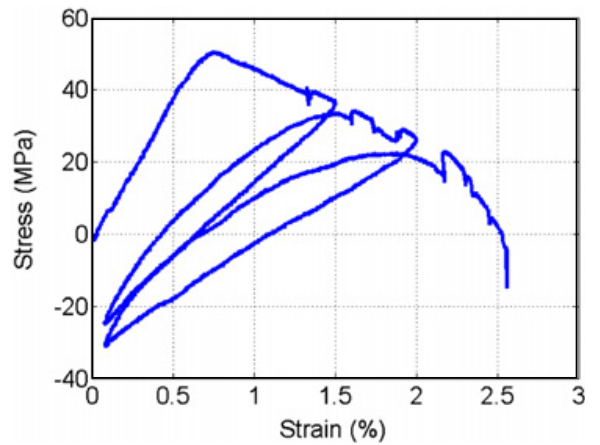

Fig. 6. The response in the transversely oriented plies from three cycles of loading and unloading of the $[0 / 90]_{6}$-laminates.

damage, indicated by the severe reduction in secant stiffness in each unloading.

\section{Coupling of yield and damage}

In the material test results presented in Figure 4, 5 and 6, both irreversible strains and reduction in secant stiffness is observed. Irreversible strains are commonly associated with plasticity and reduction in secant stiffness, with damage.

It is assumed that the longitudinal response is linear up to ultimate failure, the nonlinear shear response is caused by plasticity and that the nonlinear transverse tensile response is caused by damage. The failure criterion was adjusted not to affect any of the shear moduli, i.e. $G_{12}, G_{13}$ and $G_{23}$. The yield criterion was adjusted not

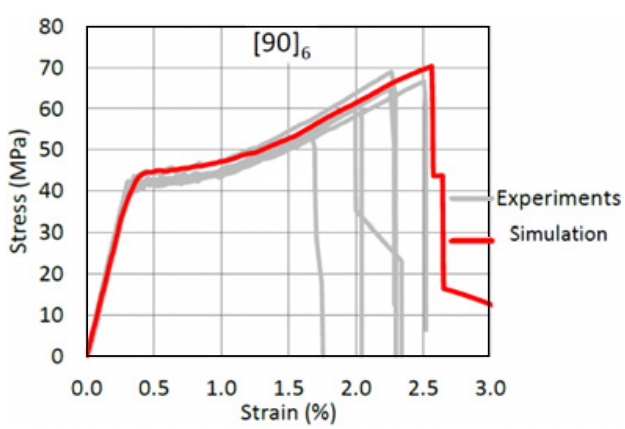

Fig. 7. Stress-strain curves from tensile loading experiments and simulation of $[90]_{6}$-laminates.

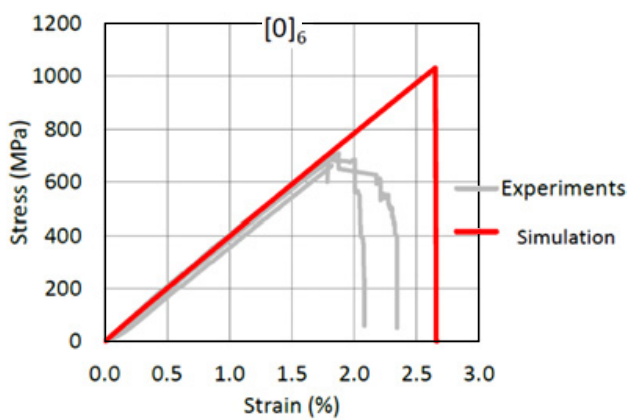

Fig. 8. Stress-strain curves from tensile loading experiments and simulation of $[0]_{6}$-laminates.

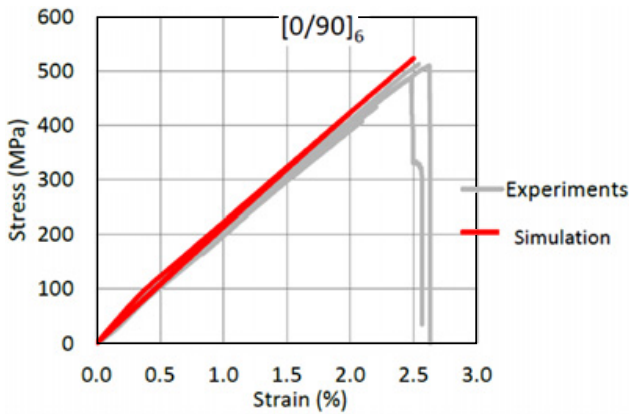

Fig. 9. Stress-strain curves from tensile loading experiments and simulation of $[0 / 90]_{6}$-laminates. The simulation is unloaded and reloaded $1 \%, 3 \%$ and $5 \%$ strain.

to affect the response in the transverse direction or in the longitudinal direction, and also, the shear response was assumed to be isotropic. The hardening curve was established based on the shear response of the ASTM D 3518 test on $[ \pm 45]_{6}$-laminates.

\section{Simulation results}

The results from the simulations are presented in Figure 7 trough 12 . In addition to the loading and unloading tests on $[0 / 90]_{6^{-}}$and $[ \pm 45]_{6}$-laminates, various laminate lay-ups have been evaluated for tensile loading and compared to experimental results. It is reasonable to assume that various combinations of plasticity and damage are present provoking the nonlinearity in the response in these laminates. 


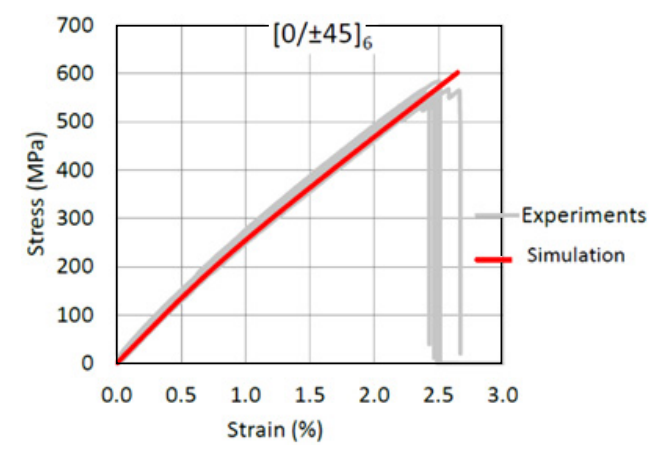

Fig. 10. Stress-strain curves from tensile loading experiments and simulation of $[0 / \pm 45]_{6}$-laminates.

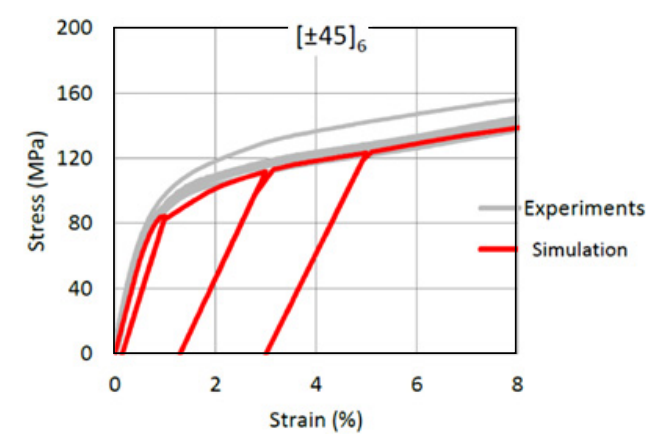

Fig. 11. Stress-strain curves from tensile loading experiments and simulation of $[ \pm 45]_{6}$-laminates. The simulation is unloaded and reloaded $1.5 \%$ and $2 \%$ strain.

\section{Discussion}

It has been shown that the presented model gives a good estimation of the nonlinear response these laminates have to tensile loading. The cyclic loading results form the simulations are in good agreement with that observed in the experimental results. This model will be further refined for use in impact modeling. In an impact-event, elastic stress waves will cause local stress oscillations which will contribute to dissipate the impact energy. When predicting the residual strength after impact, it is crucial to have a good understanding of the mechanisms involved in the dissipation of the impact energy.

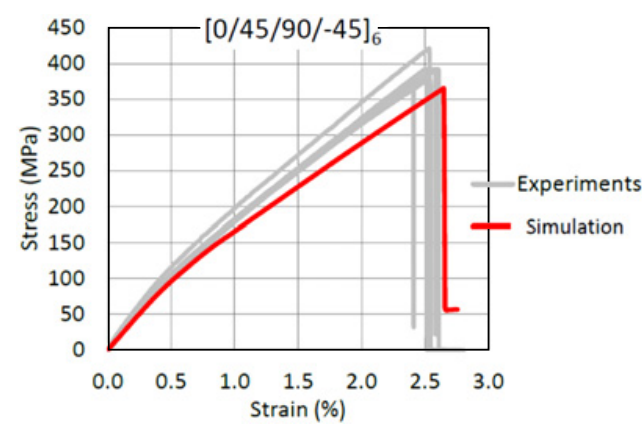

Fig. 12. Stress-strain curves from tensile loading experiments and simulation of $[0 / 45 / 90 /-45]_{6}$-laminates.

\section{Acknowledgements}

This work is part of the project "Composite structures under impact loading" financed by the Norwegian Reserch Council (193238/i40), Flowtite Technology AS, Nammo Raufoss AS and Ragasco AS. The authors would like to express their thanks for this finacial support. The authors would also like to thank the other partners in the project (NTNU, UiO and SINTEF Raufoss Manufacturing) for constructive discussions.

\section{References}

1. A. Puck and H. Schürmann, Composites Science and Technology 58, (1998) 1045-1067

2. R. Hill, Proceedings of the Royal Society of London. Series A, Mathematical and Physical Sciences 193, (1948) 281-297

3. X. Xiao, A Coupled Damage-plasticity Model for Energy Absorption in Composites

4. M.J. Hinton, A.S. Kaddour and P.D. Soden, Failure criteria in Fibre reinforced polymer composites: The World-Wide Failure Exercise (Elisvier, 2003) 1255

5. J Wiegand, N Petrinic and B Elliott, Composites Science and Technology 68, (2008) 2511-2517

6. A. Puck, J. Kopp and M. Knops, Composites Science and Technology 62, (2002) 371-378

7. A. Puck, H. Schürmann, Composites Science and Technology 62, (2002) 1633-1662 